(C) [2009] IEEE. Reprinted, with permission, from Basukala, Riyaj., Mohd Ramli Huda Adibah \& Sandrasegaran, Kumbesan. 2009, 'Performance of Well Known Packet Scheduling Algorithms in the Downlink 3GPP LTE System', Proceedings of the 2009 IEEE 9th Malaysia International Conference on Communications (MICC 2009), pp. 1-5. This material is posted here with permission of the IEEE. Such permission of the IEEE does not in any way imply IEEE endorsement of any of the University of Technology, Sydney's products or services. Internal or personal use of this material is permitted. However, permission to reprint/republish this material for advertising or promotional purposes or for creating new collective works for resale or redistribution must be obtained from the IEEE by writing to pubs-permissions@ieee.org. By choosing to view this document, you agree to all provisions of the copyright laws protecting it. 


\title{
Performance of Well Known Packet Scheduling Algorithms in the Downlink 3GPP LTE System
}

\author{
Huda Adibah Mohd Ramli ${ }^{\# 1}$, Riyaj Basukala ${ }^{\# 2}$, Kumbesan Sandrasegaran ${ }^{\# 3}$, Rachod Patachaianand ${ }^{\# 4}$ \\ ${ }^{\#}$ Faculty of Engineering and Information Technology, \\ University of Technology \\ Sydney, Australia \\ [ ${ }^{1}$ HudaAdibah.MohdRamli, ${ }^{3}$ kumbes, ${ }^{4}$ rpatacha] @eng.uts.edu.au \\ ${ }^{2}$ Riyaj.Basukala@uts.edu.au
}

\begin{abstract}
This paper investigates the performance of well known packet scheduling algorithms developed for single carrier wireless systems from a real time video streaming perspective. The performance evaluation is conducted using the downlink third generation partnership project long term evolution (3GPP LTE) system as the simulation platform. This paper contributes to the identification of a suitable packet scheduling algorithm for use in the downlink 3GPP LTE system supporting video streaming services. Simulation results show that, in the downlink 3GPP LTE system supporting video streaming services, maximum-largest weighted delay first (M-LWDF) algorithm outperforms other packet scheduling algorithms by providing a higher system throughput, supporting a higher number of users and guaranteeing fairness at a satisfactory level.
\end{abstract}

Keywords — Packet scheduling algorithms, 3GPP LTE, OFDMA, QoS, video streaming

\section{INTRODUCTION}

Long term evolution (LTE) is a new radio access technology proposed by the third generation partnership project (3GPP) in order to provide a smooth migration towards fourth generation $(4 \mathrm{G})$ wireless systems. The $3 \mathrm{GPP}$ LTE uses orthogonal frequency division multiple access (OFDMA) in the downlink. The OFDMA technology divides the available bandwidth into multiple narrow-band subcarriers and allocates a group of sub-carriers to a user based on its requirements, current system load and system configuration.

The 3GPP LTE radio network architecture consists of only one node between the user and the core network known as eNodeB which is responsible to perform all radio resource management (RRM) functions. Packet scheduling is one of the RRM functions and it is responsible for intelligent selections of users and transmissions of their packets such that the radio resources are efficiently utilized and the users' quality of service (QoS) requirements are satisfied.

Video streaming is one of the real time (RT) services that need to be supported in the 3GPP LTE system. To ensure that the QoS requirements of video streaming users are guaranteed, the packet loss rate has to be minimized by keeping it below a threshold. The packets of a video streaming user should be received by the user within its delay threshold; otherwise the packets will be discarded and hence considered as lost packets.

There are many packet scheduling algorithms developed for single carrier wireless systems being proposed in the literature [1-11]. However, the performances of these algorithms in multi-carrier wireless systems require further investigations. This paper investigates the performance of well known packet scheduling algorithms developed for single carrier wireless systems from the perspective of video streaming services. The performance evaluation is conducted using the downlink 3GPP LTE system as the simulation platform. This paper contributes to the identification of a suitable packet scheduling algorithm for use in the downlink 3GPP LTE system for supporting video streaming services.

The radio resource that is available for a user in the downlink 3GPP LTE system is defined in both frequency and time domains and is called a resource block (RB). In the frequency domain, the RB consists of 12 consecutive subcarriers ( $180 \mathrm{kHz}$ total bandwidth) and in the time domain it is made up of one time slot of $0.5 \mathrm{~ms}$ duration. A time slot consists of 7 OFDM symbols [12]. Scheduling in this system is performed at $1 \mathrm{~ms}$ interval (transmit time interval, TTI) and two consecutive RBs (in time domain) are assigned to a user for a TTI.

In this system, each user reports its instantaneous downlink channel conditions (e.g. signal-to-noise-ratio, SNR) to the serving eNodeB at each TTI. The received instantaneous downlink SNR values of each user vary on each sub-carrier and at each TTI due to the frequency-selective fading nature of multi-path propagation and time-selective fading nature due to user movement respectively $[13,14]$.

A generalized model of packet scheduling algorithm in the downlink 3GPP LTE system is given in Fig. 1. From the figure, it can be seen that, each user is assigned a buffer at the serving eNodeB. Packets arriving into the buffer are time stamped and queued for transmission based on a first-in-firstout basis. In each TTI, the packet scheduler determines which users are to be scheduled based on a packet scheduling algorithm. In this system, there is a possibility that a user may 
be allocated zero, one or more than one RBs at each TTI as shown in the figure.

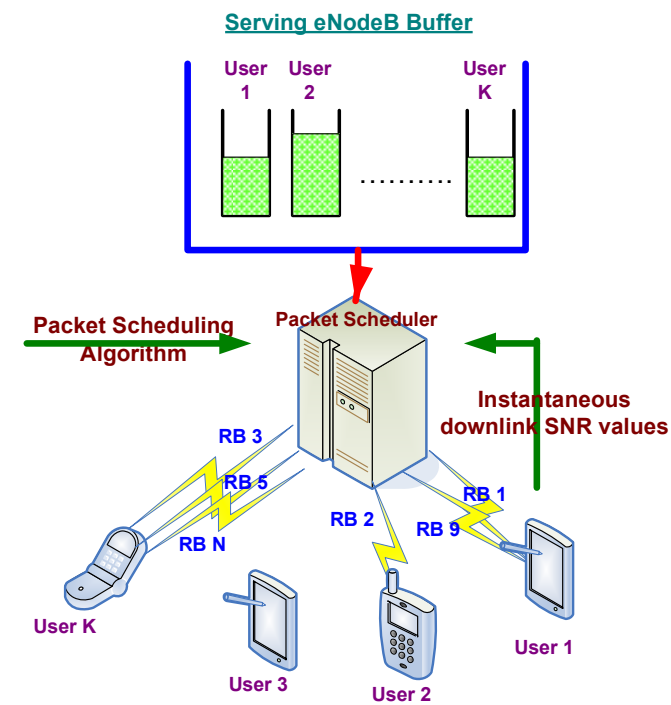

Fig. 1: Generalized Model of Packet Scheduling in the Downlink 3GPP LTE System

The remainder of this paper is organized as follows. Section II discusses about the well known packet scheduling algorithms followed by descriptions of the simulation environment in Section III. Section IV contains the results of the simulation while conclusions are given in Section V.

\section{Well KNOWn PACKET SCHEDUling AlgORITHMS}

The packet scheduling algorithms to be used in 3GPP LTE should aim to maximize throughput along with fairness. The algorithms that are going to be considered in this paper are the most common packet scheduling algorithms that were developed for single carrier wireless systems and good candidates for use in 3GPP LTE.

The maximum rate (Max-Rate) [8] algorithm always selects the user with the highest reported instantaneous downlink SNR value. This algorithm efficiently utilizes system resource as user's packets are transmitted on a radio resource with a good channel condition. On the other hand, a user with low instantaneous downlink SNR value will never get access to the available radio resource using Max-Rate algorithm. This user is deprived from using the radio resource unless the user's channel condition improves. This situation results in low fairness performance in Max-Rate algorithm.

Given that fairness has been an issue in Max-Rate algorithm, the round robin (RR) [1] algorithm was developed to address the problem. RR meets the fairness by allocating an equal share of packet transmission time to each user. However, throughput performance degrades significantly as the algorithm does not rely on the reported instantaneous downlink SNR values when determining the number of bits to be transmitted.

To provide a balance between throughput and fairness, the proportional fair (PF) [4] algorithm was proposed. PF was originally developed to support NRT service in code division multiple access high data rate (CDMA-HDR) system. Assuming $r_{i}(t)$ as the achievable data rate of user $i$, PF algorithm selects a user with the highest metric $k$ defined as follows:

$k=\arg \max \frac{r_{i}(t)}{R_{i}(t)}$

and:

$R_{i}(t)=\left(1-\frac{1}{t_{c}}\right) * R_{i}(t-1)+\frac{1}{t_{c}} * r_{i}(t-1)$

where $R_{i}(t)$ is the average data rate of user $i$ over a time window $\left(t_{c}\right)$ of an appropriate size. This update window size determines between maximizing throughput and satisfying fairness of each user in PF algorithm.

Scheduling decisions that rely on channel conditions only are insufficient to support multimedia applications due to strict delay requirements. Hence, several packet scheduling algorithms that satisfy this new requirement have been developed.

The maximum-largest weighted delay first (M-LWDF) [6] is an algorithm designed to support multiple real time data users in CDMA-HDR system. A user is selected based on the following equation:

$k=\arg \max a_{i} W_{i}(t) \frac{r_{i}(t)}{R_{i}(t)}$

and:

$a_{i}=-\frac{\left(\log \delta_{i}\right)}{\tau_{i}}$

where $W_{i}(t)$ is the head of line (HOL) packet delay (time difference between the current time and the arrival time of a packet) of user $i$ at time $t, \tau_{i}$ is the delay threshold of user $i$ and $\delta_{i}$ is the maximum probability for HOL packet delay of user $i$ to exceed the delay threshold of user $i$. M-LWDF algorithm achieves a relatively low packet loss ratio (PLR) with a good throughput and fairness performance since it incorporates HOL packet delay together with PF properties (e.g. the ratio of achievable data rate to the average data rate) when determining users' priority.

The exponential/proportional fair (EXP/PF) [2, 7] algorithm was developed to support multimedia applications in an adaptive modulation and coding and time division multiplexing (AMC/TDM) system. A user in AMC/TDM system can either belong to a RT service or a NRT service. Therefore, the EXP/PF equation to be used for a user depends on the user's service type. The metric $k$ is computed for each user for RT and NRT services using the following equation:

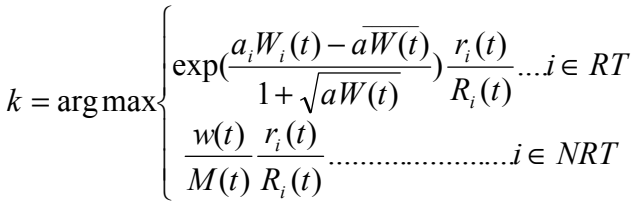


and:

$$
\begin{aligned}
& \overline{a W(t)}=\frac{1}{N_{R T}} \sum_{i \in R T} a_{i} W_{i}(t) \\
& w(t)=\left\{\begin{array}{l}
w(t-1)-\varepsilon \ldots \ldots . W_{\max }>\tau_{\text {max }} \\
w(t-1)+\frac{\varepsilon}{k} \ldots . . W_{\max }<\tau_{\text {max }}
\end{array}\right.
\end{aligned}
$$

where $M(t)$ is the average number of RT packets waiting at the serving eNodeB buffer at time $t, \varepsilon$ and $k$ are constants, $W_{\max }$ is the maximum HOL packet delay of all RT service users and $\tau_{\max }$ is the maximum delay constraint out of RT service users. In the EXP/PF algorithm, RT users receive a higher priority than NRT users when their HOL packet delays are approaching the delay deadline.

When compared with the single carrier wireless systems that only schedule one user at each scheduling instant, the multi-carrier wireless systems schedule multiple users at each scheduling instant as there are multiple groups of sub-carriers available to be shared by all users within the system at the same time. Using the downlink 3GPP LTE as the simulation platform, the user with the highest metric (of the simulated packet scheduling algorithm) on each $\mathrm{RB}$ is selected for transmission. In the downlink 3GPP LTE system, an RB that is poor for a user may be a good RB for another user, thus enabling multi-path fading effect to be exploited rather than combated in this system.

\section{SIMULATION ENVIRONMENT}

In this paper a single hexagonal cell of $5 \mathrm{MHz}$ bandwidth with $25 \mathrm{RBs}$ and $2 \mathrm{GHz}$ carrier frequency is used. The eNodeB has a fixed location at the centre of the cell and it controls all the available RBs. These RBs are to be shared by all users within the cell. The 3GPP LTE downlink system parameters are given in Table I.

TABLE I

3GPP LTE DOWNLINK SYSTEM PARAMETERS

\begin{tabular}{|cc|}
\hline Parameters & Values \\
\hline Carrier Frequency & $2 \mathrm{GHz}$ \\
\hline Bandwidth & $5 \mathrm{MHz}$ \\
\hline Number of Sub-carriers & 300 \\
\hline Number of RBs & 25 \\
\hline Number of Sub-carriers per RB & 12 \\
\hline Sub-Carrier Spacing & $15 \mathrm{kHz}$ \\
\hline Slot Duration & $0.5 \mathrm{~ms}$ \\
\hline Scheduling Time (TTI) & $1 \mathrm{~ms}$ \\
\hline Number of OFDM Symbols per Slot & 7 \\
\hline
\end{tabular}

There are $K$ (80 to 120$)$ video streaming users within the cell and they are uniformly located within the serving eNodeB. Users are constantly moving at speeds between 1$100 \mathrm{~km} / \mathrm{h}$ in random directions. A wrap-around method [15] is employed at the cell boundary to ensure users always remain within the simulation area. Several assumptions are made in this paper due to time limitations as well as in order to reduce complexity of the system simulation. These assumptions are consistent with other works [16-18].

It is assumed that each user reports its instantaneous downlink SNR values on each RB and at each TTI to the serving eNodeB and this reporting is assumed to be error-free and delay-free. The reported instantaneous downlink SNR values are determined based on sub-carrier located at the centre frequency of each RB. It is also assumed that all subcarriers are used for data transmission and equal downlink transmit power is allocated on each sub-carrier.

Pathloss [19], shadow fading [20] and multi-path fading [21] are used to determine the channel gain and hence the instantaneous downlink SNR value of each user on each RB. It is assumed in this paper that at any time instant, multi-path values vary on each $\mathrm{RB}$ whereas pathloss and shadow fading are fixed on each RB. The channel gain $\left(\operatorname{Gain}_{i, j}(t)\right)$ of user $i$ on $\mathrm{RB} j$ at time $t$ is computed using:

$\operatorname{Gain}_{i, j}(t)=10^{\left(\frac{p_{i}(t)}{10}\right)} * 10^{\left(\frac{\xi_{i}(t)}{10}\right)} * 10^{\left(\frac{m p a t h_{i, j}(t)}{10}\right)}$

where $p l_{i}(t)$ and $\xi_{\mathrm{i}}(t)$ are the pathloss and shadow fading gain of user $i$ at time $t$, respectively and mpath $_{i, j}(t)$ is the multi-path fading gain of user $i$ on $\mathrm{RB} j$ at time $t$. From the computed channel gain, the instantaneous downlink SNR value of user $i$ on RB $j$ at time $t\left(\gamma_{i, j}(t)\right)$ is computed using the approach proposed in [22] as given below:

$\gamma_{i, j}(t)=\frac{P_{\text {total }} * \operatorname{Gain}_{i, j}(t)}{N\left(I+N_{o}\right)}$

where $P_{\text {total }}$ is the total eNodeB downlink transmit power, $N$ is the number of available RBs, $N_{o}$ is the thermal noise and $I$ is the inter-cell interference.

The reported instantaneous downlink SNR value is used to determine the data rate, which is the number of bits in two consecutive RBs that a user can support at each TTI. The approach proposed in [23] is used to compute the number of bits per symbol of user $i$ at time $t$ on a sub-carrier within RB $j\left(\right.$ nbits $_{i, j}(t) /$ symbol). The user's achievable data rate $\left(d r_{i}(t)\right)$ at time $t$ on two consecutive RBs are determined using:

$d r_{i}(t)=\frac{\text { nbits }_{i, j}(t)}{\text { symbol }_{\text {slot }}} * \frac{\text { nsymbols }}{T T I} * \frac{\text { nslots }}{R B}$

where nsymbols/slot is the number of symbols per slot, $n s l o t / T T I$ is the number of slots per TTI and $n s c / R B$ is the number of sub-carriers per RB. The minimum instantaneous downlink SNR value and the associated achievable data rate used are given in Table II. 
TABLE II

INSTANTANEOUS DOWNLINK SNR TO DATA RATE MAPPING TABLE

\begin{tabular}{|ccc|}
\hline $\begin{array}{c}\text { Minimum } \\
\text { Instantaneous } \\
\text { Downlink SNR } \\
\text { Value (dB) }\end{array}$ & $\begin{array}{c}\text { Modulation and } \\
\text { Coding }\end{array}$ & $\begin{array}{c}\text { Data } \\
\text { Rate } \\
\text { (kbps) }\end{array}$ \\
\hline 1.7 & QPSK (1/2) & 168 \\
\hline 3.7 & QPSK (2/3) & 224 \\
\hline 4.5 & QPSK (3/4) & 252 \\
\hline 7.2 & 16 QAM (1/2) & 336 \\
\hline 9.5 & 16 QAM (2/3) & 448 \\
\hline 10.7 & 16 QAM (3/4) & 504 \\
\hline 14.8 & 64 QAM (2/3) & 672 \\
\hline 16.1 & 64 QAM (3/4) & 756 \\
\hline
\end{tabular}

A video streaming service of a $128 \mathrm{kbps}$ source video rate is used in the simulation and its parameters are shown in Table III. The threshold for HOL packet delay is set to $20 \mathrm{~ms}$ which is the maximum waiting time of a packet at the serving eNodeB buffer [24]. The buffer of each user is assumed to be infinite and packet is considered lost when it is discarded, not due to buffer overflow.

TABLE III

PARAMETERS OF VIDEO STREAMING SERVICES [25]

\begin{tabular}{|c|c|c|}
\hline Information types & Distribution & $\begin{array}{l}\begin{array}{c}\text { Distribut } \\
\text { ion }\end{array} \\
\text { Parameters } \\
\end{array}$ \\
\hline $\begin{array}{l}\text { Inter-arrival time } \\
\text { between the beginning of } \\
\text { successive frames }\end{array}$ & $\begin{array}{r}\text { Deterministic } \\
\text { (Based on 20fps) }\end{array}$ & $50 \mathrm{~ms}$ \\
\hline $\begin{array}{l}\text { Number of packets } \\
\text { (slices) in a frame }\end{array}$ & Deterministic & 8 \\
\hline Packet (slice) size & $\begin{array}{c}\text { Truncated } \\
\text { Pareto } \\
\text { (Mean }=50 \text { byt } \\
\text { es, } \\
\text { max }=125 \text { bytes }) \\
\end{array}$ & $\begin{array}{l}\mathrm{K}=40 \text { byte } \\
\mathrm{s}, \alpha=1.2\end{array}$ \\
\hline $\begin{array}{c}\text { Inter-arrival time } \\
\text { between packets (slices) in } \\
\text { a frame }\end{array}$ & $\begin{array}{c}\text { Truncated } \\
\text { Pareto } \\
(\text { Mean }=6 \mathrm{~ms}, \\
\text { Max }=12.5 \mathrm{~ms})\end{array}$ & $\begin{aligned} \mathrm{K} & =2.5 \mathrm{~ms}, \\
\alpha & =1.2\end{aligned}$ \\
\hline
\end{tabular}

The performance of each algorithm is evaluated based on system throughput, PLR and fairness defined as follow:

system throughput $=\frac{1}{T} \sum_{i=1}^{K} \sum_{t=1}^{T}$ ptransmit $_{i}(t)$

$$
P L R=\frac{\sum_{i=1}^{K} \sum_{t=1}^{T} \operatorname{pdiscard}_{i}(t)}{\sum_{i=1}^{K} \sum_{t=1}^{T} \operatorname{psize}_{i}(t)}
$$

fairness $=1-\frac{\text { ptotaltran }_{\text {smit }}{ }_{\max }-\text { ptotaltran } \text { smit }_{\min }}{\sum_{i=1}^{K} \sum_{t=1}^{T} \text { psize }_{i}(t)}$

where $\operatorname{ptransmit}_{i}(t), \operatorname{pdiscard}_{i}(t)$ and $\operatorname{psize}_{i}(t)$ are the size of transmitted packets, the size of discarded packets and the size of all packets that have arrived into the serving eNodeB buffer of user $i$ at time $t$, respectively, ptotaltransmit max $_{\text {ax }}$ and ptotaltransmit $_{\text {min }}$ are the total size of the transmitted packets of the most and the least served users, respectively, $K$ is the total number of users and $T$ is the total simulation time.

\section{Simulation RESUlts AND Discussions}

Fig. 2 shows system throughput of the evaluated packet scheduling algorithms. From the figure, it can be observed that M-LWDF and EXP/PF algorithms outperform Max-Rate, $\mathrm{PF}$ and RR algorithms in terms of system throughput. The RR algorithm achieves the lowest system throughput as it does not consider channel conditions when making scheduling decisions. Furthermore, a fixed data rate being assigned to all users in the simulation somewhat limits the throughput in RR from increasing beyond the saturation level as the number of users increases.

Since channel conditions are utilized when determining users' priority in M-LWDF, EXP/PF, Max-Rate and PF algorithms, this has enabled multi-user diversity to be exploited. Therefore, it can be observed in the figure that, system throughput of these algorithms increase with increasing number of users.

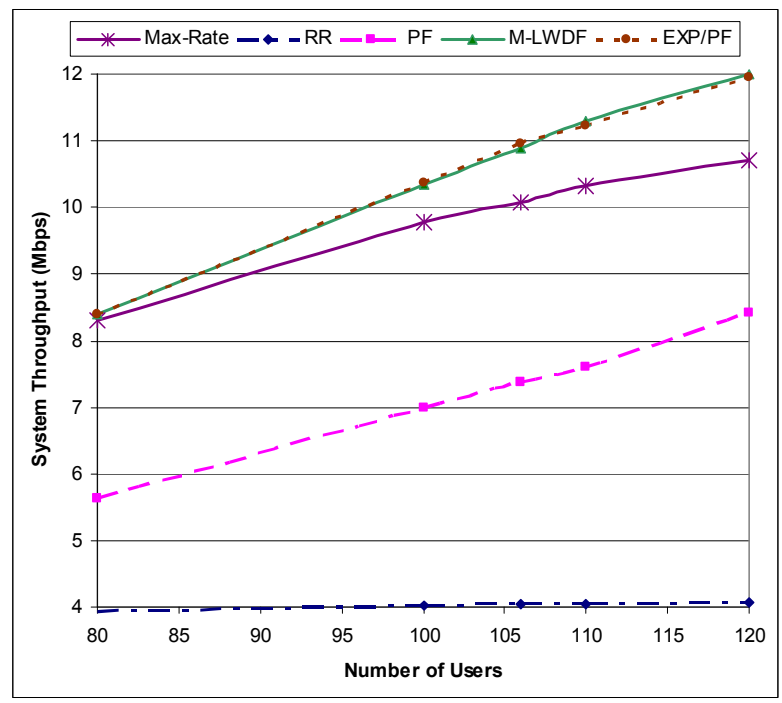

Fig. 2: System Throughput vs. Number of Users

In a video streaming service environment, it is important to maintain the PLR threshold below 1\% [26] such that the QoS requirement of video streaming service users are satisfied. In Fig. 3, it can be observed that less than 80 users can be supported by Max-Rate, PF and RR algorithms at the target PLR threshold with Max-Rate algorithm having better PLR performance than PF and RR algorithms. On the other hand, both M-LWDF and EXP/PF algorithms can support a higher number of users at $1 \%$ PLR threshold. The M-LWDF and $\mathrm{EXP} / \mathrm{PF}$ algorithms achieve significantly better PLR performance when compared with Max-Rate, PF and RR algorithms, as these algorithms consider packet delay 
information when making scheduling decisions.

It can also be observed that the M-LWDF and EXP/PF algorithms compete with each other in satisfying the QoS requirement of video streaming services. From the simulation result, we observed that approximately 106 and 110 users can be supported by M-LWDF and EXP/PF algorithms simultaneously at the target PLR threshold. Since there is a slight difference between the number of users that can be supported by M-LWDF and EXP/PF, which algorithm can best support video streaming services in the downlink 3GPP LTE system is inconclusive.

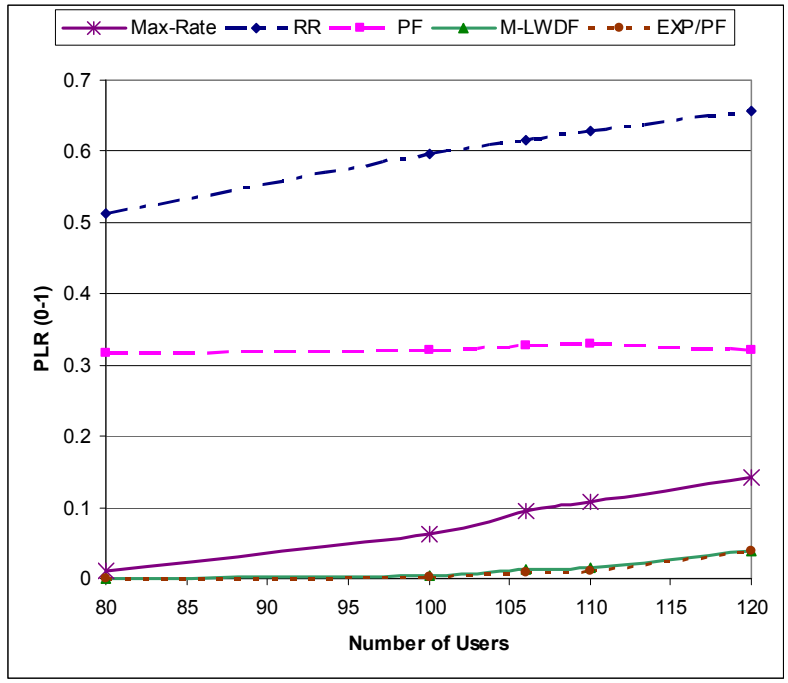

Fig. 3: Packet Loss Ratio vs. Number of Users

The fairness performance of each packet scheduling algorithm is shown in Fig. 4. In general, the RR algorithm has the best fairness performance since the algorithm allocates equal share of packet transmission time to each user as well as a fixed data rate is assigned to each user within the system.

When compared with RR algorithm, the fairness performance of M-LWDF algorithm falls slightly lower than the RR algorithm at a higher number of users and better than the RR algorithm at a lower number of users. This is a good indication that M-LWDF algorithm does satisfy fairness requirement of video streaming service users at a sufficient level. On the other hand, the fairness performance of EXP/PF algorithm degrades significantly with increasing number of users. Though the EXP/PF algorithm has a good system throughput and PLR performance, its fairness performance is very poor when compared with M-LWDF algorithm at a higher number of users.

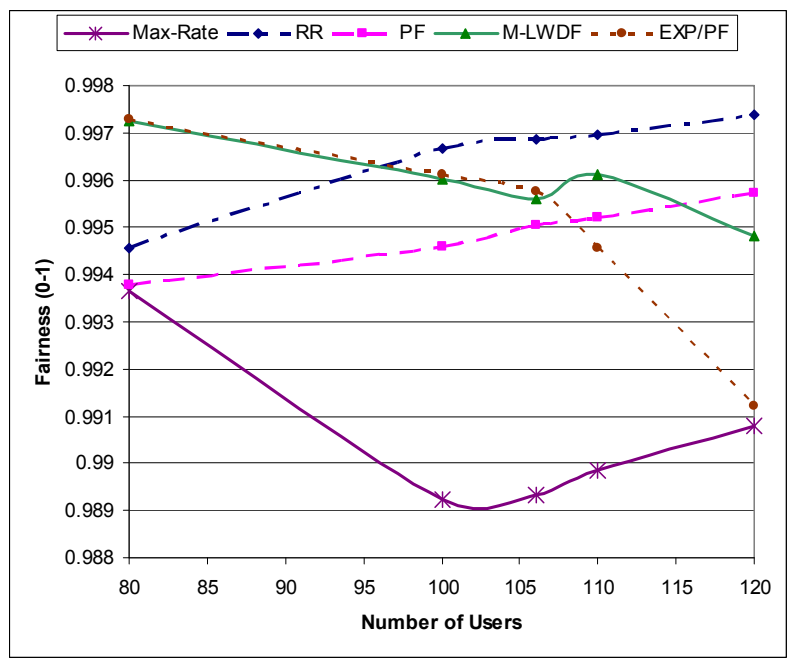

Fig. 4: Fairness vs. Number of Users

\section{CONCLUSIONS}

This paper investigates the performance of well known packet scheduling algorithms in the downlink 3GPP LTE system. It identifies the strengths and weaknesses of these well known algorithms in the downlink 3GPP LTE system such that the video streaming service requirements could be met. The simulation results show that in the downlink 3GPP LTE system supporting video streaming services, M-LWDF algorithm outperforms other packet scheduling algorithms by providing a higher system throughput, supporting a higher number of users and guaranteeing fairness at a satisfactory level. Therefore we conclude that M-LWDF is the best known candidate for packet scheduling algorithm for the downlink 3GPP LTE system that supports video streaming service environment.

\section{REFERENCES}

[1] E. Dahlman, S. Parkvall, J. Skold, and P. Beming, 3G Evolution: HSPA and LTE for Mobile Broadband, First ed.: Elsevier Ltd., 2007.

[2] J.-H. Rhee, J. M. Holtzman, and D. K. Kim, "Performance Analysis of the Adaptive EXP/PF Channel Scheduler in an AMC/TDM System," IEEE Communications Letters, vol. 8, pp. 4978-4980, Aug. 2004.

[3] V. Huang and Z. Weihua, "QoS-oriented Packet Scheduling for Wireless Multimedia CDMA Communications," IEEE Transactions on Mobile Computing, vol. 3, pp. 73-85, 2004.

[4] A. Jalali, R. Padovani, and R. Pankaj, "Data Throughput of CDMAHDR a High Efficiency-High Data Rate Personal Communication Wireless System," in IEEE 51st Vehicular Technology Conference Proceedings, Tokyo, 2000, pp. 1854-1858.

[5] M. Andrews, L. Qian, and A. Stolyar, "Optimal Utility based Multi-user Throughput Allocation subject to Throughput Constraints," in IEEE Proceedings of 24th Annual Joint Conference of the IEEE Computer and Communications Societies (INFOCOM) 2005, pp. 2415-2424 vol. 4.

[6] M. Andrews, K. Kumaran, K. Ramanan, A. Stolyar, P. Whiting, and R. Vijayakumar, "Providing Quality of Service over a Shared Wireless Link," IEEE Communications Magazine, vol. 39, pp. 150-154, Feb. 2001 .

[7] J.-H. Rhee, J. M. Holtzman, and D. K. Kim, "Scheduling of Real/Nonreal Time Services: Adaptive EXP/PF Algorithm," in The 57th IEEE Semiannual Vehicular Technology Conference. vol. 1, 2003, pp. 462466. 
[8] B. S. Tsybakov, "File Transmission over Wireless Fast Fading Downlink," IEEE Transactions on Information Theory, vol. 48, pp. 2323-2337, 2002.

[9] A. Gyasi-Agyei, "Multiuser Diversity based Opportunistic Scheduling for Wireless Data Networks," IEEE Communications Letters vol. 9, pp. 670-672, 2005

[10] S. Shakkotai and A. L. Stolyar, "Scheduling Algorithms for a Mixture of Real-Time and Non-Real-Time Data in HDR" Aug. 2000.

[11] H. Ming, Z. Junshan, and J. Sadowsky, "Traffic Aided Opportunistic Scheduling for Downlink Transmissions: Algorithms and Performance Bounds," in Twenty-third Annual Joint Conference of the IEEE Computer and Communications Societies, 2004, pp. 1652-1661 vol.3.

[12] J. Zyren, "Overview of 3GPP Long Term Evolution Physical Layer," freescale semiconductor 2007.

[13] J. Gross, H. Karl, F. Fitzek, and A. Wolisz, "Comparison of Heuristic and Optimal Subcarrier Assignment Algorithms," in Proceedings of International Conference on Wireless Networks, 2003.

[14] A. K. F. Khattab and K. M. F. Elsayed, "Opportunistic Scheduling of Delay Sensitive Traffic in OFDMA-based Wireless Networks," in International Symposium on a World of Wireless, Mobile and Multimedia Networks, 2006.

[15] A. G. Orozco Lugo, F. A. Cruz Prez, and G. Hernandez Valdez, "Investigating the Boundary Effect of a Multimedia TDMA Personal Mobile Communication Network Simulation," in IEEE VTS 54th Vehicular Technology Conference, 2001, pp. 2740-2744 vol.4.

[16] L. Xiantao, L. Guangyi, W. Ying, and Z. Ping, "Downlink Packet Scheduling for Real-Time Traffic in Multi-User OFDMA System," in IEEE 64th Vehicular Technology Conference, 2006, pp. 1 - 5
[17] N. Xu, T. Zou, Y. Wang, and P. Zhang, "A MC-GMR Scheduler for Shared Data Channel in 3GPP LTE System," in IEEE 64th Vehicular Technology Conference, 2006, pp. 1-5.

[18] A. Pokhariyal, T. E. Kolding, and P. E. Mogensen, "Performance of Downlink Frequency Domain Packet Scheduling for the UTRAN Long Term Evolution," in IEEE 17th International Symposium on Personal, Indoor and Mobile Radio Communications, 2006, pp. 1-5.

[19] D. C. Cox, R. R. Murray, and A. W. Norris, "800-MHz attenuation measured in and around suburban houses," AT\&T Bell Lab Technical Journal, vol. 63, pp. 921-954, July-Aug 1984.

[20] M. Gudmundson, "Correlation Model for Shadow Fading in Mobile Radio Systems," in Electronics Letters. vol. 27, 1991, pp. 2145-2146.

[21] C. Komninakis, "A Fast and Accurate Rayleigh Fading Simulator," in IEEE Globecom San Francisco, CA, 2003.

[22] K. Kim, G.-M. Yeo, B.-H. Ryu, and K. Chang, "Interference Analysis and Subchannel Allocation Schemes in Tri-Sectored OFDMA Systems," in IEEE 66th Vehicular Technology Conference, 2007, pp. 1857-1861.

[23] X. Qiu and K. Chawla, "On the Performance of Adaptive Modulation in Cellular Systems," in IEEE Transactions on Communications. vol. 47, 1999, pp. 884-895.

[24] L. Senthikumar and V. Sankaranayanan, "QoS Provisioning Through a Delay Based Endpoint Admission Control for Diffserv Network," Information Technology Journal, vol. 3, pp. 448-453, 2006.

[25] 3GPP2, "cdma2000 Evaluation Methodology," December 102004.

[26] T. Janevski, Traffic Analysis and Design of Wireless IP Networks. Norwood, MA: Artech House, 2003. 